\title{
The effect of age on task-related modulation of interhemispheric balance
}

\author{
P. Talelli $\cdot$ W. Waddingham $\cdot$ A. Ewas $\cdot$ \\ J. C. Rothwell · N. S. Ward
}

Received: 21 August 2007/ Accepted: 29 October 2007/Published online: 27 November 2007

(C) The Author(s) 2007

\begin{abstract}
Normal aging is associated with less lateralised task-related activation of the primary motor cortices. It has been hypothesized, but not tested, that this phenomenon is mediated transcallosaly. We have used Transcranial Magnetic Stimulation to look for age-related changes in interhemispheric inhibition (IHI). Thirty healthy individuals (aged 19-78 years) were studied using a paired-pulse protocol at rest and during a low-strength isometric contraction with the right hand. The IHI targeting the right motor cortex was assessed at two intervals, $10 \mathrm{~ms}$ (IHI10) and $40 \mathrm{~ms}$ (IHI40). The corticospinal excitability of the left hemisphere was assessed by means of input-output curves constructed during voluntary construction. Age was not correlated with IHI10 or IHI40 at rest. During muscle contraction IHI tended to increase at both intervals. However, this increase in IHI during the active condition (changeIHI) was less evident with advancing age for the $40 \mathrm{~ms}$ interval $(r=0.444, P=0.02)$; in fact a degree of disinhibition was often present. There was no correlation between age and changeIHI10. Age was negatively correlated with the area under the recruitment curve $(r=-0.585$,
\end{abstract}

P. Talelli $(\bowtie) \cdot$ J. C. Rothwell

Sobell Department of Motor Neuroscience and Movement

Disorders, Institute of Neurology, University College London,

Queen SQ, Box 146, London WC1N 3BG, UK

e-mail: p.talelli@ion.ucl.ac.uk

W. Waddingham · A. Ewas · N. S. Ward

Department of Brain Repair and Rehabilitation,

Institute of Neurology, University College London,

London WC1N 3BG, UK

N. S. Ward

Wellcome Trust Centre for Neuroimaging, Institute of Neurology, University College London, London WC1N 3BG, UK
$P=0.001)$ and the size of the maximum MEP collected $(r=-0.485, P=0.007)$. ChangeIHI and measures of corticospinal excitability were not intercorrelated. In conclusion, task-related increases in interhemispheric inhibition seem to diminish with advancing age. This phenomenon is specific for long-latency IHI and may underlie the age-related bihemispheric activation seen in functional imaging studies. The mechanism underlying changes in IHI with advancing age and the association with changes in corticospinal excitability need further investigation.

Keywords Motor system - Interhemispheric inhibition · Transcranial magnetic stimulation · Aging

\section{Introduction}

Physical performance declines with increasing age (Hackel et al. 1992; Smith et al. 1999). Although age-related changes in muscular (Vandervoort 2002) and neural architecture (Dorfman and Bosley 1979; Haug and Eggers 1991; Madden et al. 2004) are an important cause of this decline, there is increasing interest in the role of functional alterations occurring throughout the motor system. Excitation-contraction uncoupling (Delbono et al. 1995), motor unit remodelling (Wang et al. 1999), changes in the agonist-antagonist activation pattern (Hortobagyi and Devita 2006) and reorganization of the central motor pathways (Kido et al. 2004; Ward and Frackowiak 2003; Minati et al. 2007) are all areas currently under study. Understanding the mechanisms underlying these phenomena will aid differentiation between inevitable functional failure and potential compensatory strategies which in turn may allow the development strategies to combat this age-related decline (Delbono 2003). 
Functional imaging has been used to assess age-dependent changes in the cerebral motor system in humans (for review see Ward 2006). In general, motor task-related brain activation is seen in a wider network with advancing age. The exact pattern of age-related change depends on the task being performed. However, it has been repeatedly shown that in older individuals there is increased bihemispheric activation during the execution of motor tasks that in younger individuals involve mainly lateralized processing. In particular, with advancing age there is less deactivation of the primary motor cortex (M1) ipsilateral to the moving hand (Ward and Frackowiak 2003; Naccarato et al. 2006; Ward et al. 2007). It has been suggested, but not tested, that the underlying mechanism for this phenomenon involves changes in interhemispheric connections between the motor cortices.

Transcranial magnetic stimulation (TMS) can be used to study interhemispheric connectivity between the motor cortices. At rest, this connectivity consists primarily of an inhibitory effect (interhemispheric inhibition, IHI) with a latency of 6-50 ms (Ferbert et al. 1992; Di Lazzaro et al. 1999; Daskalakis et al. 2002; Chen 2004). This tonic IHI is modulated and follows a specific time course during preparation and execution of voluntary movement (Murase et al. 2004; Duque et al. 2005, 2007). During the execution of an isometric muscle contraction, the IHI (at $10 \mathrm{~ms}$ latency) targeting the M1 ipsilateral to the moving hand has been shown to increase (Ferbert et al. 1992). This "extra" inhibition is in keeping with the primarily unilateral activation pattern seen in imaging studies of movement and has been interpreted as a means of minimizing mirror activity (Leocani et al. 2000; Duque et al. 2005). Taskrelated changes in longer latency IHI, i.e. $40 \mathrm{~ms}$, have not been studied in detail but there is some evidence that they may be distinct to the ones seen in short latency IHI (Chen et al. 2003).

Previous TMS studies have shown that there is reduction of activity in the intracortical inhibitory circuits in the elderly (Peinemann et al. 2001; Hortobagyi et al. 2006). It has been suggested that reduced inhibition represents a mechanism to compensate for the deleterious effects of aging on several levels of the motor system including the primary motor cortex (Pitcher et al. 2003; Oliviero et al. 2006) but these notions remain mainly hypothetical (Wassermann 2002).

The main purpose of this study was to investigate whether normal aging has an effect on the task-related modulation of IHI measured at two different latencies, 10 and $40 \mathrm{~ms}$. We hypothesized that with increasing age there would be less extra activation in the inhibitory circuits targeting the M1 ipsilateral to the moving hand. In order to gain some insight of the mechanisms and the physiological meaning of these changes we also performed measures of corticospinal excitability on the side contralateral to the moving hand and looked for correlations with age and IHI measures.

\section{Methods}

Subjects

Thirty healthy right-handed volunteers (mean age 42.9 years, range $19-78 ; 30 \%$ female) participated in the study after giving informed consent. They reported no history of neurological illness, psychiatric history, vascular disease or hypertension and they were not taking regular medication. The study was approved by local Ethics Committee.

\section{Transcranial magnetic stimulation}

Subjects were seated in an armchair with their eyes open. EMGs were recorded via $\mathrm{Ag} / \mathrm{AgCl}$ electrodes placed over the First Dorsal Intersosseus (FDI) bilaterally, using a belly-tendon montage. Signals were filtered $(30 \mathrm{~Hz}$ to $10 \mathrm{kHz}$ ), amplified using a Digitimer 360 (Digitimer Ltd, Welwyn Garden City, Herts., UK) and stored on computer via a Power 1401 data acquisition interface (Cambridge Electronic Design Ltd, Cambridge, UK). Analysis of data was carried out using Signal Software (Cambridge Electronic Design).

Two figure-of-eight coils connected to two monophasic Magstim 200 stimulators were used for the experiments (all Magstim Co., UK). A 70-mm coil was used for motor hot spot identification and threshold measurements on both sides of the brain. The motor hotspot was defined as the scalp location where TMS consistently resulted in the largest MEP. The resting motor threshold (RMT) was defined as the lowest intensity needed to evoke an EMG response of $50 \mu \mathrm{V}$ in $50 \%$ of the trials with the FDI relaxed; the active motor threshold (AMT) was defined as the intensity which evoked a $200 \mu \mathrm{V}$ EMG response in $50 \%$ of the trials with a background FDI contraction of $10-15 \%$ of the maximum voluntary contraction (MVC). For all studies requiring activation of the FDI, visual feedback was provided using an oscilloscope.

A recruitment curve (RC) for the active MEP amplitude elicited in the left FDI was obtained using the $70 \mathrm{~mm}$ coil while the subjects maintained a background FDI contraction of 15-20\% MVC. Ten MEPs were collected and averaged at the following stimulus intensities: 90, 100, 110, 120, 140, 150, 160 and 170\% AMT. The contraction level was tested by measuring the mean value of the rectified EMG in the $80 \mathrm{~ms}$ preceding the TMS pulse. The averaged 
peak-to-peak amplitude of the unrectified MEPs was then expressed as a ratio of the maximum peak-to-peak amplitude of the unrectified compound action potential (CMAP) evoked by supramaximal electrical stimulation of the ulnar nerve at the wrist using a Digitimer pulse stimulator (model DS7).

Interhemispheric inhibition (IHI) was measured using a standardised paired-pulse paradigm (Ferbert et al. 1992). IHI is expressed as the reduction in the response to a suprathreshold TMS pulse (test) delivered to the M1 when another suprathreshold pulse is delivered to the contralateral M1 6-50 ms earlier (conditioning). Here, we concentrated on the IHI targeting the right M1. Hence the conditioning pulse was given over the left M1 using the same $70-\mathrm{mm}$ coil. For the test pulse a smaller $50 \mathrm{~mm}$ figure-of-eight coil was held over the right M1; this allowed us to accommodate two coils on the head of all the subjects without compromising the exact positioning of the coils over the motor hot-spot (Ferbert 1992). MEPs were measured from the left FDI. Ten single (test) and ten paired-pulse (conditioning + test) trials were randomly intermingled and averaged. IHI was defined as the conditioned/test MEP amplitude ratio, smaller values reflecting stronger IHI. Interstimulus intervals (ISIs) of 10 and $40 \mathrm{~ms}$ were studied in different blocks. IHI was initially measured at rest (rest $\mathrm{IHI} 10$ and rest $\mathrm{IHI} 40$ ). The stimulation intensity for both the conditioning and the test stimuli was adjusted to evoke an MEP of $1-1.5 \mathrm{mV}$ in the contralateral FDI muscle. For the active condition (activeIHI10 and activeIHI40) the subjects were instructed to contract the right FDI to 15-20\% of their MVC in response to an auditory cue preceding the conditioning stimulus by $600 \mathrm{~ms}$. In this way, we made sure that the subject had reached the target level of background contraction for at least $200 \mathrm{~ms}$ before the conditioning pulse.
This was to avoid measuring changes in IHI that are associated with the preparation of the movement rather than a steady isometric contraction (Duque et al. 2005, 2007). EMG activity in the left FDI was also recorded and contaminated trials were rejected to avoid the confounding effect of mirror activity. The stimulation intensity both for the conditioning and test stimulus was the same as in the resting state, as in previous studies (Ferbert et al. 1992). The absolute values of activeIHI were then expressed as a ratio to the values at rest for the respective ISI (changeIHI10 and changeIHI40). ChangeIHI therefore reflects the change seen in the IHI targeting the right motor cortex when the right hand is active. Values $<1$ reflect stronger inhibition, while values $>1$ reflect less inhibition.

\section{Statistical analysis}

Analysis was performed using SPSS v.14 (SPSS Inc., USA). Exploratory plotting and statistical tests (ShapiroWilk) confirmed normal distribution for most variables. Some of the variables were skewed by a single outlier and this could not be corrected by logarithmic transformations; the outlier was therefore discarded from the dataset to allow use of parametric tests.

Data obtained during the RC paradigm were plotted against the stimulation intensity. In most subjects the resulting curves were sigmoidal in shape. Two representative examples, one from a young and one from an old subject, are shown in Fig. 1. The area under the RC (AUC) was calculated using the method of trapezoid integration to provide a summary measure of the corticospinal output across all stimulation intensities. Data points were then fitted in the Boltzmann sigmoidal model
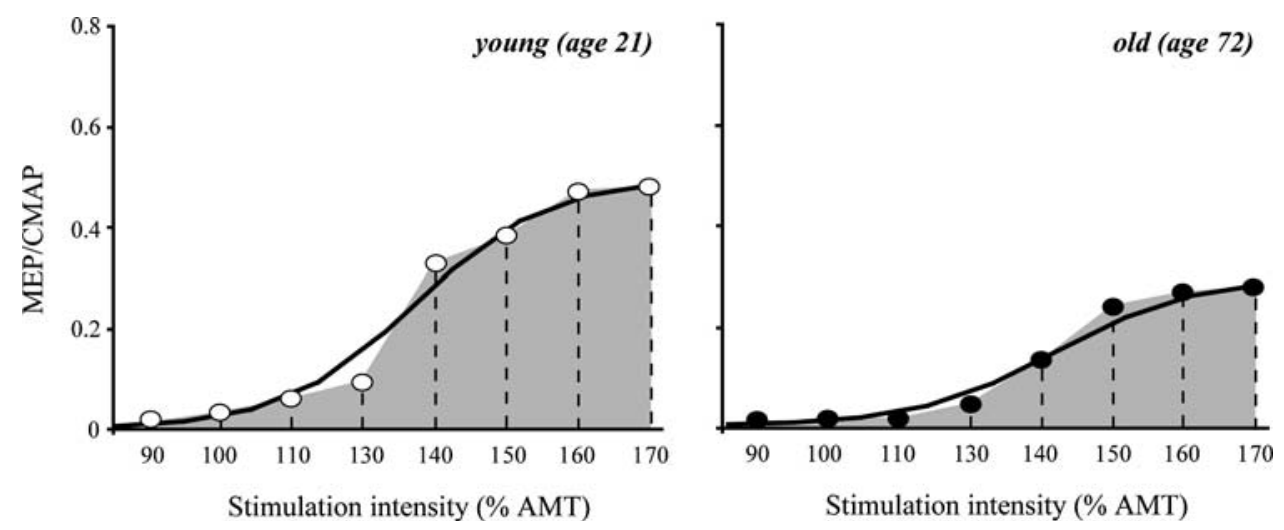

Fig. 1 Representative examples of the recruitment curves plotting the amplitude of the active MEPs against the stimulation intensity. The circles represent the collected data. The line represents the relation predicted by the Boltzmann model. The area under the curve

(grey area) was calculated with the method of trapezoid integration using the actual data collected during the construction of the curves. CMAP, compound motor action potential, AMT, active motor threshold 
based on the modified Levenberg-Marquardt nonlinear least-mean-squares algorithm (Press et al. 1986). This function is often used to provide parameter estimates for RCs (Capaday et al. 1999; Devanne et al. 1997). The data were best fitted by a 4-parameter equation in which the amplitude of the MEP at a given stimulation intensity (I) is estimated as: $\mathrm{MEP}=\mathrm{MEP}_{\min }+\left(\mathrm{MEP}_{\max }-\mathrm{MEP}_{\min }\right) /$ $1+\exp ((150-I) /$ slope $) \cdot \mathrm{MEP}_{\max }$ and $\mathrm{MEP}_{\min }$ represent the maximum and minimum MEP amplitude, respectively; I50 represents the stimulation intensity required to get a response $50 \%$ of the maximum. The inverse of the slope parameter (1/slope) is directly proportional to the maximal steepness of the curve, which occurs at I50 (Devanne et al. 1997). The $R^{2}$ co-efficient of determination was greater than 0.89 in all subjects.

Pair-wise comparisons between different TMS measures were performed using paired $t$ tests. Correlations with age and between TMS measures were assessed by computing Pearson's product-moment correlation coefficient. We have also looked for correlations between these parameters and gender. Partial and part correlations were employed as appropriate. Significance level was set at $P<0.05 . \mathrm{v}$

\section{Results}

The mean and range of the values for all TMS measures are summarized in the Table 1.

\section{Corticospinal excitability}

Threshold values were not different between the left and right M1. Age was not correlated with RMT or AMT on either side or the amplitude of the CMAP. The mean amplitude (CMAP corrected) of the active MEPs recorded during the construction of the recruitment curves was negatively correlated with age at all stimulation intensities between 100-160\%AMT (100\%AMT: $r=-0.431$, $P=0.02 ; 110 \%$ AMT: $r=-0.397, P=0.03 ; 120 \%$ AMT: $r=-0.481, \quad P=0.007 ; \quad 140 \%$ AMT: $\quad r=-0.556$, $P=0.001 ; 150 \%$ AMT: $r=-0.457, P=0.11 ; 160 \%$ AMT: $r=-0.463, P=0.01)$. The same was true for the amplitude of the maximum MEP recorded (Pearson's $r=$ $-0.485, P=0.007$ ) (Fig. 2a). Age was negatively correlated with the total area under the RC (Pearson's $r=$ $-0.585, P=0.001$ ) (Fig. 2b). From the parameters estimated using the Boltzmann model, MEPmax showed a weak correlation with age $(r=-0.397, P=0.04)$; age was not correlated with the maximum slope or I50.

Gender was not correlated with any of the TMS measures of corticospinal excitability.
Table 1 TMS measures of corticospinal excitability and interhemispheric inhibition

\begin{tabular}{|c|c|c|}
\hline \multicolumn{3}{|l|}{ Corticospinal excitability } \\
\hline & $\begin{array}{l}\text { Left } \\
\text { hemisphere }\end{array}$ & $\begin{array}{l}\text { Right } \\
\text { hemisphere }\end{array}$ \\
\hline \multicolumn{3}{|c|}{ Motor thresholds (\% stimulator's intensity) } \\
\hline Resting (RMT) & $37.6(28-65)$ & $36.4(29-50)$ \\
\hline Active (AMT) & $28.4(19-45)$ & $28(22-39)$ \\
\hline \multicolumn{3}{|c|}{$R C$ of active MEP amplitude (CMAP corrected) } \\
\hline \multicolumn{3}{|l|}{ Mean MEP amplitude recorded } \\
\hline $90 \%$ AMT & $0.02(0.01-0.06)$ & - \\
\hline $100 \%$ AMT & $0.03(0.01-0.07)^{*}$ & - \\
\hline $110 \%$ AMT & $0.06(0.02-0.18)^{*}$ & - \\
\hline $120 \%$ AMT & $0.09(0.03-0.35)^{*}$ & - \\
\hline $140 \%$ AMT & $0.25(0.06-0.51)^{*}$ & - \\
\hline $150 \%$ AMT & $0.30(0.10-0.57)^{*}$ & - \\
\hline $160 \%$ AMT & $0.34(0.13-0.56)^{*}$ & - \\
\hline $170 \%$ AMT & $0.22(0.39-0.62)$ & - \\
\hline $\begin{array}{l}\text { Maximum MEP amplitude } \\
\text { recorded }\end{array}$ & $0.38(0.13-0.62)^{*}$ & - \\
\hline AUC & $12.8(1.8-23.4)^{*}$ & - \\
\hline \multicolumn{3}{|c|}{ Parameter estimates (Boltzman model) } \\
\hline MEPmax/CMAP & $0.4(0.13-0.72)^{*}$ & - \\
\hline I50 (\%AMT) & $137(115-168)$ & - \\
\hline 1/slope & $0.14(0.05-0.37)$ & - \\
\hline \multicolumn{3}{|l|}{ IHI (targeting the right M1) } \\
\hline & $10 \mathrm{~ms}$ & $40 \mathrm{~ms}$ \\
\hline rest $\mathrm{IHI}$ & $0.61(0.32-0.9)$ & $0.6(0.28-1.1)$ \\
\hline activeIHI & $0.54(0.1-0.89)$ & $0.57(0.2-1)$ \\
\hline changeIHI (activeIHI/restIHI) & $0.96(0.42-2.47)$ & $0.97(0.5-1.49)^{*}$ \\
\hline
\end{tabular}

CMAP, compound motor action potential; AUC, area under the curve; $\mathrm{MEP}_{\max }$, maximum MEP amplitude estimated; I50, stimulation intensity needed to get a response of $50 \%$ of the maximal MEP; IHI, interhemispheric inhibition; restIHI, IHI measured with both hands relaxed; active IHI, IHI measured during a tonic contraction of the dominant hand at 15-20\% MVC; changeIHI, values $<1$ indicate stronger IHI at the active condition

* Significant correlation with advancing age $(P<0.05)$

Interhemispheric inhibition

Most subjects showed some amount of IHI at rest; only in one subject could rest IHI40 not be elicited. Paired-samples $t$ test did not show any difference between restIHI10 and restIHI40 $(P=0.44)$. Because the amount of IHI depends on the intensity used for both the conditioning and the test stimulus (Ferbert et al. 1992; Chen et al. 2003) we looked for correlations between these values and age. Age was not correlated with the intensities used to stimulate either the left M1 (70-mm coil; $45.7 \pm 7.1$ for IHI10 and $45.8 \pm 7.2$ for IHI40) or the right M1 (50-mm coil; $46.2 \pm 8.6$ for 
Fig. 2 Correlations between age and TMS parameters. There was a significant negative correlation between the amplitude of the maximum MEP recorded during the construction of the RC (a) and the total area under the RC (b). Age was negatively correlated with change IHI at $40 \mathrm{~ms}(\mathbf{d})$ but not at $10 \mathrm{~ms}$ (c). CMAP, compound motor action potential, RC, recruitment curve constructed during tonic contraction of the target muscle, IHI, interhemispheric inhibition, rest $\mathrm{IHI}$, IHI measured with both hands relaxed, activeIHI, IHI measured during a tonic contraction of the dominant hand at $15-20 \%$ MVC, change IHI, values $<1$ indicate stronger IHI at the active condition
A Maximum MEP amplitude

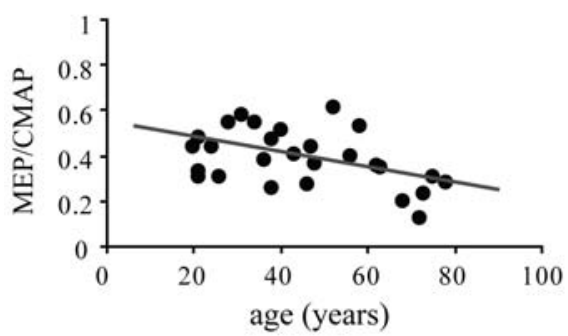

C changeIHI at 10 msec

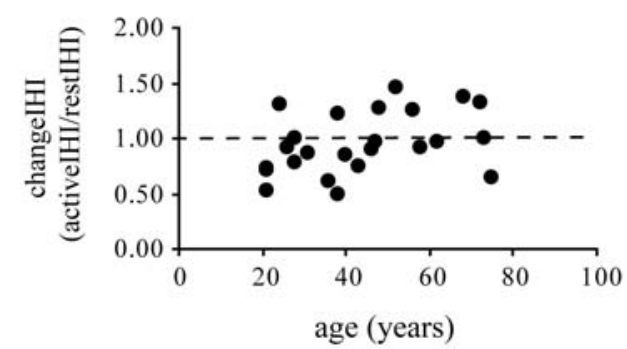

B Area under the RC

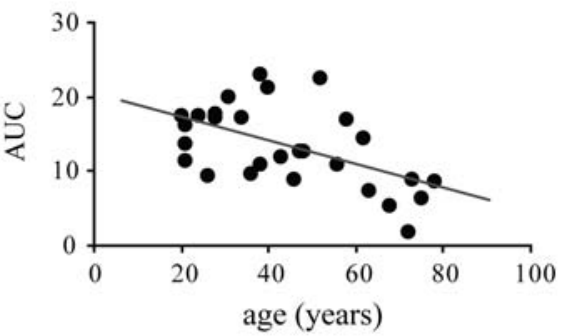

D changeIHI at $40 \mathrm{msec}$

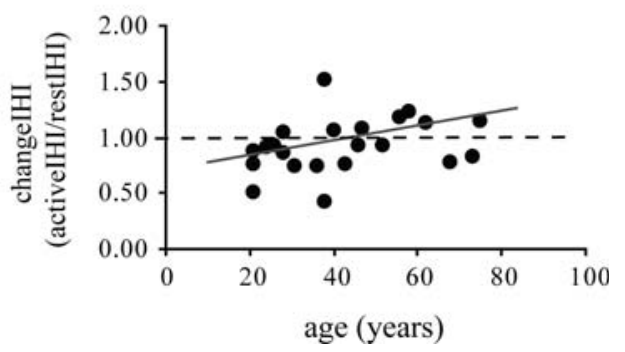

IHI10 and $46.9 \pm 8.1$ for IHI40). The amplitude of the MEPs elicited by the test stimulus and by the conditioning stimulus was slightly higher for the rest IHI40 but the values fell within the target range of $1-1.5 \mathrm{mV}$ (see below).

During activation of the right hand the MEP elicited by the test stimulus in the resting left FDI tended to increase (from $1.23 \pm 0.53$ to $1.44 \pm 0.67$ for IHI10 and from $1.48 \pm 0.58$ to $1.64 \pm 0.70$ for IHI40), but this was not significant across the group, as reported earlier for lowstrength isometric contractions (Liepert et al. 2001). As expected, the amplitude of the MEP elicited by the conditioning pulse in the right FDI increased significantly during activation of the muscle (from $1.27 \pm 0.40$ to $5.95 \pm 1.69, P<0.001$ for IHI10 and from $1.5 \pm 0.47$ to $6.17 \pm 2.57, P<0.001$ for IHI40).

There was an overall tendency for stronger IHI during activation of the right FDI at both ISIs, but this did not reach significance. In fact, changeIHI was quite variable ranging from more inhibition to less inhibition compared to the resting condition. Because changeIHI might be biased by the amount of IHI at rest we looked for correlations between changeIHI and restIHI; there was none for either ISI.

There was no correlation between age and changeIHI10 (Fig. 2c). However, we found a significant positive correlation between age and change IHI40 $(r=0.444, P=0.02)$ (Fig. 2d). In other words, when younger individuals activated their right hand the amount of inhibition targeting the ipsilateral (right) motor cortex increased; with increasing age this phenomenon was gradually attenuated and often reversed. Because age showed a significant correlation with the amplitude of the active MEPs we performed semi-partial correlations correcting for the change seen in the amplitude of the conditioning MEP between the resting and the active condition (change $\mathrm{MEP}=$ active $\mathrm{MEP} /$ resting $\mathrm{MEP}$ ). Again no correlation was found between age and changeIHI10; the correlation between age and changeIHI40 remained and was in fact slightly stronger $(r=0.553$, $P=0.006)$.

Gender was not significantly correlated with any of the IHI measures; in line with a previous report (De Gennaro et al. 2004) there was a tendency for a positive correlation between female gender and the amount of restIHI10 targeting the right hemisphere, but this finding did not reach significance in our group of subjects $(r=345, P=0.07)$.

Finally, changeIHI was not correlated with any of the measures of corticospinal excitability elicited from the stimulation of the left M1.

\section{Discussion}

The purpose of this study was to investigate the effect of age on the interhemispheric balance between the two primary motor cortices and in particular the modulation of inhibition targeting the right M1 during activation of the right hand (changeIHI). We found that the amount of tonic inhibition (rest IHI) was very variable across the group but age could not explain this variability. However, age was significantly correlated with changeIHI. As reported previously, when young individuals perform a low strength grip, the inhibition targeting the ipsilateral M1 tends to increase (Ferbert et al. 1992). Our data show that with 
increasing age this extra inhibition is not as strong and some degree of disinhibition may be present instead. Interestingly, the effect of age was significant for the $40 \mathrm{~ms}$ ISI (changeIHI40) but no correlation was found between age and changeIHI10.

Our results on changeIHI are in keeping with the findings from functional imaging studies. A number of studies have consistently shown that task-related activation is less lateralised in older individuals (for review see Ward 2006). This bilateral recruitment includes primary and non-primary motor areas; activation of the latter seems to characterise more complex tasks. Regarding the primary motor cortices, reduced deactivation of the M1 ipsilateral to the moving hand has been a common finding among studies using different scanning paradigms (Hutchinson et al. 2002; Ward and Frackowiak 2003; Naccarato et al. 2006; Ward et al. 2007). It has been hypothesized that this phenomenon could be mediated transcallosally. In support of this hypothesis, we have directly demonstrated that there is task-related reduction in interhemispheric inhibition with advancing age at least when the right hand is activated.

The differential effect of age on changeIHI10 and changeIHI40 is an interesting finding that adds to the current knowledge of the cortical circuits that these measures reflect. So far, it is thought that both short latency IHI (8-12 ms) and long latency IHI (40-50 ms) are mediated through excitatory callosal projecting neurons that are distinct from corticospinal neurones (Lee et al. 2007). In accordance with this view, corticospinal output and IHI measures in this study did not correlate. The callosal neurons are thought to activate inhibitory interneurons in the contralateral M1 (Daskalakis et al. 2002; Chen 2004), but these connections may differ for short and long latency IHI (Chen et al. 2003; Kukaswadia et al. 2005). The neurotransmitters involved are not entirely understood but there is evidence of significant involvement of $\mathrm{GABA}_{\mathrm{B}}$ ergic activity. However, Irlbacher et al. (2007), have recently demonstrated that exogenous enhancement of $\mathrm{GABA}_{\mathrm{B}}$ activity strengthens long IHI but has little effect on short IHI at rest. Long IHI was also sensitive to $\mathrm{GABA}_{\mathrm{A}}$ agonists (Irlbacher et al. 2007). Finally, Chen et al. (2004), has shown that activation of the target muscle results in a reduction in the IHI targeting the active M1 (the opposite direction than the one studied here) measured at $8 \mathrm{~ms}$ but has little effect on the IHI40. We have shown that advancing age has an effect on the modulation of IHI during voluntary muscle contraction at $40 \mathrm{~ms}$ but not at $10 \mathrm{~ms}$. We have thus provided additional evidence that IHI10 and IHI40 may respond differently to dynamic changes within the motor system and thus may represent physiologically distinct phenomena. This evidence could be strengthened in the future by testing the effect of age on
IHI in both directions, i.e. the IHI targeting the motor cortices ipsilateral and contralateral to the moving hand.

Why is IHI40 not enhanced during voluntary contraction in older individuals? One possible explanation is agerelated failure of the involved pathways. MRI studies have reported age-related reduced fractional anisotropy within the corpus callosum suggesting callosal fibre degeneration (for review see Minati et al. 2007). However, for callosal degeneration to be the cause of our finding, one would expect some effect on IHI10 as well. Another possibility is that the inhibitory circuits with which the callosal fibres synapse are less excitable. Previous studies have provided some evidence of age-related reductions in cortical inhibition. For example, $\mathrm{GABA}_{\mathrm{A}}$-ergic short-interval intracortical inhibition (SICI) assessed with a biphasic stimulator (Peinemann et al. 2001) and cortical reciprocal inhibition (Hortobagyi et al. 2006) are reduced in older individuals. However, Wasserman et al. (2002) failed to show a correlation between SICI and advancing age in a large series of normal subjects. Others have found increased SICI in middle-aged adults (Kossev et al. 2002). Further experiments, which include active conditions are needed to study these associations in the aging brain. Finally, it should be noted that although TMS-measured IHI is thought to reflect mainly transcallosal connections, Gerloff et al. (1998) has demonstrated a role for other pathways involving subcortical and/or spinal structures, the exact nature of which is unclear. Age related changes in these pathways may therefore also contribute to the correlations that we have observed.

Another question is whether the age-related reduced activity reported in multiple inhibitory systems, including IHI is an inevitable consequence of neurodegeneration or in fact represents a compensation strategy of the aging brain. Similar changes have been described in response to brain injury and have been interpreted as markers of cortical reorganization (Ward and Cohen 2004; Talelli et al. 2006). The relative preservation of changeIHI10 could suggest that the release from inhibition is not uncontrolled or at least it occurs in a hierarchical manner. For example, IHI10 may be important for direct suppression of mirror activity in the contralateral M1 (Duque et al. 2005). Longer latency IHI40, on the other hand, may reflect polysynaptic pathways extending even beyond the primary motor cortices. In that case relatively less inhibition in an active state could allow bilateral recruitment if that was necessary. In this study, there was no correlation between changeIHI40 (or any other IHI measure) and any of the measures of corticospinal excitability despite the fact that the latter appeared to be compromised in older individuals. This finding could mean that changeIHI40 does not reflect an adaptation to the failing corticospinal pathways. However, it should be kept in mind that if compensatory mechanisms 
are indeed operating with increasing age (or decreasing corticospinal output) TMS may not measure the real deficit but the compensated deficit. The next reasonable step could be to study a series of elderly individuals known to have different levels of manual dexterity and look for correlations between electrophysiological and behavioural measures, including mirror movements during performance of complex motor tasks.

We found variable effects of age on parameters relating to corticospinal excitability. Motor thresholds were not affected in keeping with previous reports (Wassermann 2002; Oliviero et al. 2006; Hortobagyi et al. 2006). We have found negative correlations between age and the amplitude of the active MEP at most stimulation intensities, between age and the amplitude of maximum MEP and finally between age and the total area under the recruitment curve (AUC). These results are in agreement with previous findings of reduced amplitude of the MEPs elicited during an isometric contraction (Oliviero et al. 2006; Sale and Semmler 2005). The values derived from the Boltzmann model did not add extra information, since there was no correlation between age and the maximal slope of the curve or the stimulation intensity required to obtain 50\% MEPmax (I50). Pitcher et al. (2003) have previously reported that I50 significantly increased with increasing age. In other words, the recruitment of the MEPs was slower at low stimulation intensities. The authors discussed that this finding may reflect reduced excitability or asynchronous activation of spinal motorneurons. In this study we did not perform measures of spinal excitability mainly due to time limitations. We have, however, constructed our RCs during background activation of the target muscle; that means that some $a$-motorneurons are already activated and the resulting measures are thus less subject to changes in spinal cord excitability (Day et al. 1989). We propose that increasing age does have a detrimental effect on the total output of primary motor cortex and that the mechanisms underlying (or compensating for) this effect are variable; thus a summary variable such as the AUC might be more suitable as a surrogate marker of overall corticospinal output.

In conclusion, this study has shown for the first time that there is an age-related reduction in the extra inhibition targeting the right hemisphere during an isometric handgrip with the right hand. These changes may underlie the bihemispheric pattern of activation seen in functional imaging studies of older individuals performing a unimanual hand task. Should our findings be confirmed, studies of interhemispheric balance may prove to be a useful marker of reorganization in the aging brain.

Open Access This article is distributed under the terms of the Creative Commons Attribution Noncommercial License which permits any noncommercial use, distribution, and reproduction in any medium, provided the original author(s) and source are credited.

\section{References}

Capaday C, Lavoie BA, Barbeau H, Schneider C, Bonnard M (1999) Studies on the corticospinal control of human walking. I. Responses to focal transcranial magnetic stimulation of the motor cortex. J Neurophysiol 81:129-139

Chen R (2004) Interactions between inhibitory and excitatory circuits in the human motor cortex. Exp Brain Res 154:1-10

Chen R, Yung D, Li JY (2003) Organization of ipsilateral excitatory and inhibitory pathways in the human motor cortex. J Neurophysiol 89:1256-1264

Daskalakis ZJ, Christensen BK, Fitzgerald PB, Roshan L, Chen R (2002) The mechanisms of interhemispheric inhibition in the human motor cortex. J Physiol 543:317-326

Day BL, Dressler D, Maertens dN, Marsden CD, Nakashima K, Rothwell JC, Thompson PD (1989) Electric and magnetic stimulation of human motor cortex: surface EMG and single motor unit responses. J Physiol 412:449-473

De Gennaro L, Bertini M, Pauri F, Cristiani R, Curcio G, Ferrara M, Rossini PM (2004) Callosal effects of transcranial magnetic stimulation (TMS): the influence of gender and stimulus parameters. Neurosci Res 48:129-137

Delbono O (2003) Neural control of aging skeletal muscle. Aging Cell 2:21-29

Delbono O, O'Rourke KS, Ettinger WH (1995) Excitation-calcium release uncoupling in aged single human skeletal muscle fibers. J Membr Biol 148:211-222

Devanne H, Lavoie BA, Capaday C (1997) Input-output properties and gain changes in the human corticospinal pathway. Exp Brain Res 114:329-338

Di Lazzaro V, Oliviero A, Profice P, Insola A, Mazzone P, Tonali P, Rothwell JC (1999) Direct demonstration of interhemispheric inhibition of the human motor cortex produced by transcranial magnetic stimulation. Exp Brain Res 124:520-524

Dorfman LJ, Bosley TM (1979) Age-related changes in peripheral and central nerve conduction in man. Neurology 29:38-44

Duque J, Mazzocchio R, Dambrosia J, Murase N, Olivier E, Cohen LG (2005) Kinematically specific interhemispheric inhibition operating in the process of generation of a voluntary movement. Cereb Cortex 15:588-593

Duque J, Murase N, Celnik P, Hummel F, Harris-Love M, Mazzocchio R, Olivier E, Cohen LG (2007) Intermanual Differences in movement-related interhemispheric inhibition. J Cogn Neurosci 19:204-213

Ferbert A, Priori A, Rothwell JC, Day BL, Colebatch JG, Marsden CD (1992) Interhemispheric inhibition of the human motor cortex. J Physiol 453:525-546

Gerloff C, Corwell B, Chen R, Hallett M, Cohen LG (1998) The role of the human motor cortex in the control of complex and simple finger movement sequences. Brain 121:1695-1709

Hackel ME, Wolfe GA, Bang SM, Canfield JS (1992) Changes in hand function in the aging adult as determined by the Jebsen test of hand function. Phys Ther 72:373-377

Haug H, Eggers R (1991) Morphometry of the human cortex cerebri and corpus striatum during aging. Neurobiol Aging 12:336-338

Hortobagyi T, Devita P (2006) Mechanisms responsible for the ageassociated increase in coactivation of antagonist muscles. Exerc Sport Sci Rev 34:29-35

Hortobagyi T, del Olmo MF, Rothwell JC (2006) Age reduces cortical reciprocal inhibition in humans. Exp Brain Res 171:322-329 
Hutchinson S, Kobayashi M, Horkan CM, Pascual-Leone A, Alexander MP, Schlaug G (2002) Age-related differences in movement representation. Neuroimage 17:1720-1728

Irlbacher K, Brocke J, Mechow JV, Brandt SA (2007) Effects of $\operatorname{GABA}(\mathrm{A})$ and $\mathrm{GABA}(\mathrm{B})$ agonists on interhemispheric inhibition in man. Clin Neurophysiol 118:308-316

Kido A, Tanaka N, Stein RB (2004) Spinal excitation and inhibition decrease as humans age. Can J Physiol Pharmacol 82:238-248

Kossev AR, Schrader C, Dauper J, Dengler R, Rollnik JD (2002) Increased intracortical inhibition in middle-aged humans; a study using paired-pulse transcranial magnetic stimulation. Neurosci Lett 333:83-86

Kukaswadia S, Wagle-Shukla A, Morgante F, Gunraj C, Chen R (2005) Interactions between long latency afferent inhibition and interhemispheric inhibitions in the human motor cortex. J Physiol 563:915-924

Lee H, Gunraj C, Chen R (2007) The effects of inhibitory and facilitatory intracortical circuits on interhemispheric inhibition in the human motor cortex. J Physiol 580:1021-1032

Leocani L, Cohen LG, Wassermann EM, Ikoma K, Hallett M (2000) Human corticospinal excitability evaluated with transcranial magnetic stimulation during different reaction time paradigms. Brain 123(Pt 6):1161-1173

Liepert J, Dettmers C, Terborg C, Weiller C (2001) Inhibition of ipsilateral motor cortex during phasic generation of low force. Clin Neurophysiol 112:114-121

Madden DJ, Whiting WL, Huettel SA, White LE, MacFall JR, Provenzale JM (2004) Diffusion tensor imaging of adult age differences in cerebral white matter: relation to response time. Neuroimage 21:1174-1181

Minati L, Grisoli M, Bruzzone MG (2007) MR spectroscopy, functional MRI, and diffusion-tensor imaging in the aging brain: a conceptual review. J Geriatr Psychiatry Neurol 20:3-21

Murase N, Duque J, Mazzocchio R, Cohen LG (2004) Influence of interhemispheric interactions on motor function in chronic stroke. Ann Neurol 55:400-409

Naccarato M, Calautti C, Jones PS, Day DJ, Carpenter TA, Baron JC (2006) Does healthy aging affect the hemispheric activation balance during paced index-to-thumb opposition task? An fMRI study. Neuroimage 32:1250-1256
Oliviero A, Profice P, Tonali PA, Pilato F, Saturno E, Dileone M, Ranieri F, Di Lazzaro V (2006) Effects of aging on motor cortex excitability. Neurosci Res 55:74-77

Peinemann A, Lehner C, Conrad B, Siebner HR (2001) Age-related decrease in paired-pulse intracortical inhibition in the human primary motor cortex. Neurosci Lett 313:33-36

Pitcher JB, Ogston KM, Miles TS (2003) Age and sex differences in human motor cortex input-output characteristics. J Physiol 546:605-613

Press WH, Flannery BP, Teukolsky SA, Vetterling WT (1986) Numerical recipes. Cambridge University Press, Cambridge

Sale MV, Semmler JG (2005) Age-related differences in corticospinal control during functional isometric contractions in left and right hands. J Appl Physiol 99:1483-1493

Smith CD, Umberger GH, Manning EL, Slevin JT, Wekstein DR, Schmitt FA, Markesbery WR, Zhang Z, Gerhardt GA, Kryscio RJ, Gash DM (1999) Critical decline in fine motor hand movements in human aging. Neurology 53:1458-1461

Talelli P, Greenwood RJ, Rothwell JC (2006) Arm function after stroke: neurophysiological correlates and recovery mechanisms assessed by transcranial magnetic stimulation. Clin Neurophysiol 117:1641-1659

Vandervoort AA (2002) Aging of the human neuromuscular system. Muscle Nerve 25:17-25

Wang FC, de Pasqua V, Delwaide PJ (1999) Age-related changes in fastest and slowest conducting axons of thenar motor units. Muscle Nerve 22:1022-1029

Ward NS (2006) Compensatory mechanisms in the aging motor system. Ageing Res Rev 5:239-254

Ward NS, Frackowiak RS (2003) Age-related changes in the neural correlates of motor performance. Brain 126:873-888

Ward NS, Cohen LG (2004) Mechanisms underlying recovery of motor function after stroke. Arch Neurol 61:1844-1848

Ward NS, Swayne OB, Newton JM (2007) Age-dependent changes in the neural correlates of force modulation: An fMRI study. Neurobiol Aging June 11

Wassermann EM (2002) Variation in the response to transcranial magnetic brain stimulation in the general population. Clin Neurophysiol 113:1165-1171 